\title{
Unstable Resonators with Gosper-Island Boundary Conditions: Virtual-Source Computation of Fractal Eigenmodes
}

\author{
J. M. Christian ${ }^{1}$, J. G. Huang ${ }^{2}$ \\ 1. Joule Physics Laboratory, University of Salford, Greater Manchester M5 4WT, United Kingdom \\ 2. Faculty of Computing, Engineering and Science, University of South Wales, Pontypridd CF37 1DL, United Kingdom
}

The Gosper island is a well-known fractal belonging to a family of self-similar "root 7" curves constructed from a simple iterative algorithm [1]. One begins with a regular hexagon (the initiator, corresponding to iteration $n=0$ ) with sides of reference length $l_{0}$, and then breaks each of these straight-edge elements into three equal segments of length $l_{n}=l_{0}\left(1 / 7^{1 / 2}\right)^{n}$ where $n=1,2,3, \ldots$ (the generator stages). If the total number of length elements after applying the generator $n$ times is given by $N_{n}=6 \times 3^{n}$, then the Hausdorff-Besicovich dimension of such a curve is calculated to be $D \equiv \lim _{n \rightarrow \infty}-\log \left(N_{n}\right) / \log \left(l_{n}\right)=2 \log (3) / \log (7) \approx 1.1292$.

In this presentation, we report on our latest theoretical results predicting the modes of unstable resonators $[2,3]$ when the small feedback mirror has a shape corresponding to increasing iterations of the Gosper island fractal. A fully two-dimensional generalization of Southwell's virtual source (2D-VS) method [4] (itself an approximation of Horwitz's asymptotic theory [5]) is deployed, whereby the resonator is unfolded into an equivalent sequence of apertures illuminated by a plane wave. Each aperture has a characteristic size (capturing a band of pattern spatial scalelengths), and it acts as a virtual source of diffracted waves that are computed using edge-wave decompositions within a circulation-integral method [6]. The empty-cavity eigenmodes are then constructed from a linear combination of the constituent single-aperture Fresnel patterns.
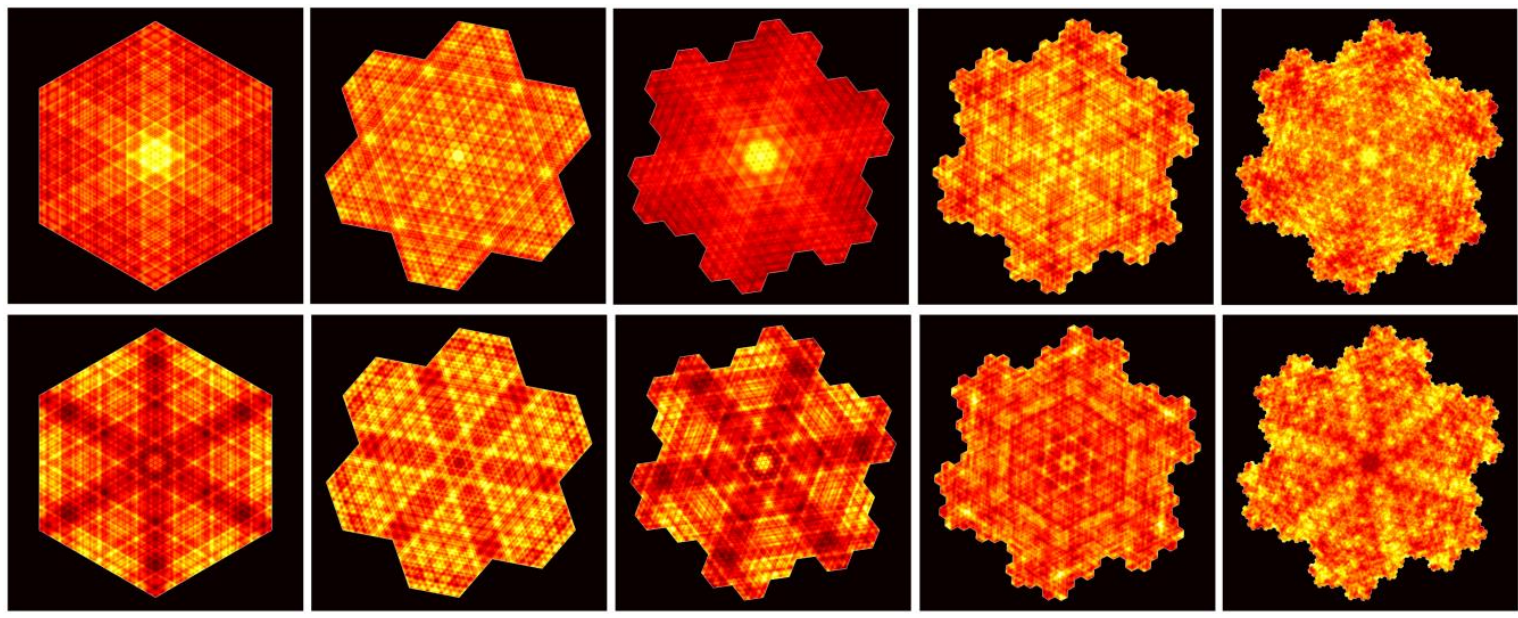

Fig. 1. 2D-VS computations of the mode patterns for an unstable resonator (parameters: $N_{\text {eq }}=30$ and $M=1.5$ ) based on the Gosper island curve whose feedback mirror progresses through the first four applications of the generator algorithm (left to right). Top row: lowest-loss modes. Bottom row: next-lowest-loss modes.

Unstable resonators are described by two key physical parameters: the round-trip magnification $M$ and the equivalent Fresnel number $N_{\mathrm{eq}}[3,4]$. A systematic review will be given of fractal eigenmode patterns for Gosper-island resonators. Spectra will be presented for the cavity eigenvalues $\left\{\alpha_{m}\right\}$ with $m=0,1,2, \ldots$, whose magnitudes and arguments prescribe round-trip losses and phase shifts, respectively, as functions of $M$ and $N_{\text {eq }}$. The weighting factors in the 2D-VS expansions are parametrized by the individual eigenvalues, and so our method gives direct access to a hierarchy of higher-order modes after only a single application (see Fig. 1). In contrast, conventional computational schemes based on fast Fourier transforms and ABCD (paraxial) matrix optics tend to yield only the lowest-loss modes (with higher-order modes often proving awkward to excite). We conclude with a summary of results for dimension estimations, where specialist software [7] has been used to quantify various different measures [8] including roughness-length, rescaled range, and variogram.

\section{References}

[1] B. B. Mandelbrot, The Fractal Geometry of Nature, Freeman (1982).

[2] G. P. Karman et al., "Fractal modes in unstable resonators," Nature 402, 138 (1999).

[3] G. H. C. New et al., "Diffractive origin of unstable resonator modes," Opt. Commun. 193, 261 (2001).

[4] W. H. Southwell, "Virtual-source theory of unstable resonator modes," Opt. Lett. 6, 487 (1981).

[5] P. Horwitz, "Asymptotic theory of unstable-resonator modes," J. Opt. Soc. Am. 63, 1528 (1973).

[6] J. G. Huang et al., "Fresnel diffraction and fractal patterns from polygonal apertures," J. Opt. Soc. Am. A 23, 2768 (2006).

[7] BENOIT 1.3, TruSoft International Inc. www.trusoft-international.com.

[8] J. Brewer and L. Di Girolamo, "Limitations of fractal dimension estimation algorithms with implications for cloud studies," Atmos. Res. 82, 433 (2006) 